\title{
Slow-release artificial tear inserts in the treatment of dry eyes in patients with rheumatoid arthritis
}

\author{
JOHN C HILL \\ From the Departments of Ophthalmology, Groote Schuur Hospital, and University of Cape Town
}

SUMMARY Twenty-two patients with rheumatoid arthritis and moderate to severe bilateral keratoconjunctivitis sicca were treated with artificial tears and a soluble slow-release artificial tear insert in a crossover trial. While using the inserts all but two patients reported an improvement in their symptoms. All the patients showed an improvement in the degree of conjunctival and corneal staining while using the inserts $(\mathrm{p}=0.0001$ and 0.00001 respectively). Nineteen patients $(86 \%)$ found the inserts an acceptable form of therapy and $14(64 \%)$ preferred them to other treatment regimens. Placement of the insert was not a problem despite all the patients having rheumatoid changes in their hands resulting in varying degrees of deformity. Two patients experienced initial difficulties with placement, but these were quickly resolved.

The treatment of patients with keratoconjunctivitis sicca (KCS) remains a problem. Various types of treatment are used to augment tear function. Occlusion of the puncta helps to prevent drainage of tears, but usually supplementation with artificial tears is necessary. Numerous preparations are available, and by trial and error the one most suitable for a given patient can be found. The most popular preparations are based on methylcellulose or polyvinyl alcohol, and act by replacing the aqueous component of tears and by helping to stabilise the tear film. The disadvantage of this mode of therapy is that it is short lived and drops must be instilled regularly, especially in severely affected patients. No study has satisfactorily shown that the solutions remain in contact with the cornea for more than 15 minutes after instillation. ${ }^{12}$ This problem becomes significant in patients with rheumatoid arthritis (RA) in whom manual dexterity is reduced. KCS is common in these patients, the usual reported incidence being between 15 and $25 \%,{ }^{34}$ but higher rates have been found. ${ }^{5}$ In a study of 104 RA patients in this hospital ${ }^{6} 22$ patients $(21 \cdot 2 \%)$ were found to have evidence of KCS as shown by ocular symptoms and corneal or conjunctival staining, together with a reduced tear break-up time (BUT), reduced No. 1 Schirmer test, or poor marginal tear strip.

Correspondence to Dr J C Hill, FRCS, Department of Ophthalmology, Medical School, Observatory 7925, Cape Town, South Africa.
A number of workers have investigated the use of various types of slow-release artificial tears (SRAT). The inserts were found to increase BUT and to stabilise the tear film, both in animals ${ }^{7}$ and in normal human volunteers. ${ }^{89}$ SRAT have been reported to be beneficial in patients with $\mathrm{KCS}^{10-14}$ and in dry eyes secondary to the practolol syndrome. ${ }^{15}$ In these studies there was an improvement in both subjective symptoms and in the objective evaluation of corneal and conjunctival staining with rose Bengal. Two problems encountered were expulsion of the insert and blurring of vision resulting from the thickened precorneal tear film. These problems were overcome to a great extent by making the inserts smaller. ${ }^{91112} \mathrm{~A}$ commercially available rod shaped SRAT insert is now available and is supplied individually packeted (Merck Sharp and Dohme). The insert is composed of preservative-free hydroxypropyl cellulose, weighs $5 \mathrm{mg}$, and measures approximately $3 \times 1 \mathrm{~mm}$. One insert is placed into the inferior conjunctival fornix each morning by means of a soft flexible applicator. Fluid is imbibed, possibly from the conjunctival tissue, ${ }^{13}$ and the insert swells to form a small soft mass. The hydroxypropyl cellulose is released continuously into the tear film. One insert usually lasts all day; it completely dissolves and does not require removal. The absence of preservatives in the inserts may be an added advantage, as many of these substances are reported to cause irritation and instability of the tear film. ${ }^{12}$ 
The use of SRAT would be ideal for patients who, because of deformity or the necessity of frequent instillation of drops, find it difficult to keep their eyes adequately moist. However, placement of the insert requires a certain degree of manual dexterity. Difficulty in placing the insert may therefore prevent older patients and those with RA and hand deformity from using this form of therapy. Little mention has been made of this problem in previous studies, but Katz et al. ${ }^{12}$ reported that one patient with severe RA and one with bilateral aphakia dropped out of their trial because of difficulty with insertion. Other studies of SRAT in patients with KCS have included patients in whom the disorder had a wide range in severity and various causes.

This paper reviews a group of RA patients with varying degrees of hand deformity and moderate to severe KCS, who received methylcellulose drops and SRAT inserts in a crossover trial.

\section{Patients and methods}

Twenty-two patients (19 females and 3 males) with RA and bilateral KCS were recruited into the study. Their average age was 50.7 years (range 39 to 66 years), and the mean duration of KCS symptoms was 5.6 years (range 23 months to 19 years). All were using artificial tears frequently, and most had been referred from the arthritis clinic in this hospital for ophthalmological assessment. A full eye examination was performed and a patient was admitted into the study if all the following criteria were fulfilled in both eyes: symptoms of KCS despite the use of artificial tears; a Schirmer's test (without anaesthesia) of $5 \mathrm{~mm}$ or less; a BUT of 10 seconds or less; corneal staining with $1 \%$ rose Bengal stain.

An attempt was made to assess the effect of SRAT on the patients' symptoms and on the degree of epithelial damage as indicated by staining. These two criteria were thought to represent the most important subjective and objective criteria respectively in KCS.

At the initial examination patients were questioned about their symptoms and asked to grade them as absent, mild, moderate, or severe. These were scored $0,1,2$, or 3 respectively. The conjunctiva and cornea were stained with $1 \%$ rose Bengal by applying a microdrop to the superior bulbar conjunctiva. The amount of staining was assessed as absent, mild, moderate, or severe and scored $0,1,2$, or 3 respectively. The initial examination was performed while the patients were using their regular tear replacement therapy.

Certain criteria excluded patients from this study. These were: any ocular infection, trauma, or surgery within the preceding three months; the use of contact lenses; the presence of other ocular diseases except lens opacities; or the use of any ocular medication except tear replacements.

The patients were allocated randomly according to a computer-generated random numbers table into two groups. The first group used a single soluble insert each morning. This was placed into the inferior conjunctival fornix with a flexible applicator. The second group used methylcellulose artificial tears, 2 drops four times a day. After two weeks the patients were reassessed, and both groups then used artificial tears for a week. The original treatment was then switched so that group 2 used the insert daily for two weeks and group 1 continued on artificial tears.

At the end of each trial period the patients were fully examined and their symptoms and staining were graded as described above. On completion of the period on the inserts the patients were asked about any difficulties in placing the insert and whether it was stable or extruded. At the end of the study the patients were asked whether they preferred using drops or the inserts.

\section{Results}

All 22 patients had RA with varying degrees of hand deformity and loss of dexterity. Two patients had initial difficulty in placing the inserts into the lower fornix but overcame the problem after a few attempts. Six patients experienced some difficulty in retaining the inserts, but in four this did not persist. The two remaining patients lost their inserts in the afternoon and continued with drops until another insert was used the next morning. In total, eight patients $(36 \%)$ experienced some difficulty in using the insert, either with placement or retention, but this persisted in only two patients $(9 \%)$. No serious side effects were encountered during the trial.

The median scores of the patient's symptoms on both the insert and the drops are shown in Table 1. Significance was calculated by the Wilcoxon test with Bonferonni correction. Because the eyes of a patient

Table 1 Median scores of symptoms: left eye (22 eyes)

\begin{tabular}{llll}
\hline Symptom & $\begin{array}{l}\text { Drops } \\
(\text { semi-IQR) }\end{array}$ & $\begin{array}{l}\text { Inserts } \\
(s e m i-I Q R)\end{array}$ & $\begin{array}{l}\text { Significance }(p) \\
\text { left eye }(\text { right eye) }\end{array}$ \\
\hline Burning & $1.23(0.595)$ & $0.15(0.324)$ & $0.0005(0.0002)$ \\
Tearing & $0.23(0.467)$ & $0.72(0.611)$ & $0.12^{*}(0.31)^{*}$ \\
Dryness & $1.28(0.667)$ & $0.08(0.289)$ & $0.0005(0.0002)$ \\
Itching & $0.94(0.690)$ & $0.15(0.324)$ & $0.0013(0.0005)$ \\
Photophobia & $0.15(0.324)$ & $0.08(0.289)$ & $0.39 *(0.39)^{*}$ \\
Soreness & $0.83(0.628)$ & $0.15(0.324)$ & $0.0043(0.003)$ \\
Blurring & $0.02(0.261)$ & $0.02(0.261)(*)$ \\
Foreign body & $1.1(0.367)$ & $0.19(0.370)$ & $0.0003(0.00001)$ \\
\hline
\end{tabular}

*Not significant.

Semi-IQR =semi-interquartile range (or quartile deviation). 
Table 2 Median scores or rose Bengal staining: left eye (22 eyes)

\begin{tabular}{llll}
\hline & $\begin{array}{l}\text { Drops } \\
(\text { semi-IQR) }\end{array}$ & $\begin{array}{l}\text { Inserts } \\
(\text { semi-IQR) }\end{array}$ & $\begin{array}{l}\text { Significance }(p) \\
\text { left eye }(\text { right eye })\end{array}$ \\
\hline Conjunctiva & $1 \cdot 23(0 \cdot 858)$ & $0 \cdot 11(0 \cdot 306)$ & $0 \cdot 0001(0.00001)$ \\
Cornea & $1 \cdot 27(0 \cdot 869)$ & $0 \cdot 15(0.324)$ & $0.00001(0.00001)$ \\
\hline
\end{tabular}

Semi-IQR: see footnote to Table 1.

cannot be considered independent,${ }^{16}$ only the results for each patient's left eye are given $(n=22)$. The results for the right eyes were similar, but only the significance levels are tabulated. There was a significant improvement in burning, dryness, itching, soreness, and foreign body sensation while they were using the inserts. The degree of blurring was the same with both types of treatment. There was a small increase in tearing and decrease in photophobia. Neither change was statistically significant. All but two patients reported improvements in their symptoms while using the inserts.

Table 2 shows the median scores for conjunctival and corneal staining. There was a significant improvement in these signs while the patients were using the inserts, and all patients improved on this therapy.

At the end of the trial the patients were asked which form of therapy they preferred. Fourteen (64\%) preferred the insert; these included two patients who had had initial problems in retaining the insert. Eight patients preferred drops as their main form of therapy, though five stated a wish to have SRAT inserts for intermittent use. The main reasons given for preferring drops were the difficulty in placing the insert (three patients), less blurring with drops (three patients), and the cooling effect of drops (six patients). The degree of hand deformity had no correlation with the type of therapy preferred. Four patients with gross hand deformity were among the most enthusiastic supporters of the insert, whereas two patients with mild hand deformity experienced difficulty in placement.

\section{Discussion}

Slow-release delivery units for ocular medication have not met with much success. Problems with extrusion and discomfort have been encountered, and most attempts have been abandoned. Unnoticed loss of an antiglaucoma system can have serious consequences, and inspection of the fornices can itself precipitate extrusion of the unit. SRAT are designed to alleviate symptoms of KCS, and loss of the insert will result in recurrence of symptoms, thus alerting the patient to the loss. Only two patients
$(9 \%)$ in this study had persistent problems with extrusion of the inserts; this usually occurred during the afternoon after many hours of useful therapy. Loss of the insert was obvious to the patient, but in cases of doubt the insert can be palpated through the skin of the lower lid and felt to be present in the lower fornix.

To become a useful treatment SRAT inserts must be easy to use by the patients they are designed to help. Placement of the inserts is a potential problem that could limit this type of therapy to certain groups of patients. RA patients with secondary hand deformities would be expected to highlight this difficulty. However, few problems of this nature were found in this study. Only two patients experienced initial difficulty in placement and both overcame the problem. Both these patients had minimal hand deformities.

Our experience with normal controls has shown that inserts are poorly tolerated and are soon expelled. This is not the case in patients with KCS, who, because of their symptoms, seem better able to tolerate a foreign body in their fornices. This tolerance is reinforced by the improvement in symptoms that occurs with the insert in situ. Only two patients considered their overall symptoms worsened while on SRAT. As in other studies, a significant improvement in most symptom scores was reported by our patients. Unlike other studies, however, a problem with blurring while on SRAT was not found, and although a higher median score for tearing while on SRAT was recorded this was not significant. Other studies ${ }^{10-12}$ that reported decreased vision with the inserts did not make corneal staining with rose Bengal a criterion for inclusion into their study. It is possible that in this study any deleterious effect on vision from the insert was masked by the improvement in the integrity of the corneal epithelium and subsequent improvement in the visual acuity. All patients showed improvement of conjunctival and corneal staining while on the SRAT; the changes in the median scores were highly significant.

Fourteen $(64 \%)$ of the patients stated a preference for SRAT and a further five requested them for intermittent use. Thus a total of 19 patients $(86 \%)$ indicated some degree of approval for this form of therapy. Many patients wished to use drops if they felt a need, the usual reason given being their 'cooling effect'. Subsequent follow-up has not revealed any problems arising from this dual therapy; in particular the insert does not seem to be more prone to expulsion. Other studies ${ }^{101114}$ reported a very similar percentage of patients who preferred SRAT as the main form of therapy, though in the study by Katz et al. ${ }^{12} 75 \%$ of their patients preferred inserts. The similar preference rate in this study indicates that no 
serious or specific problems were encountered in the use of SRAT inserts by RA patients.

In conclusion, this study demonstrates the usefulness of SRAT in the management of KCS. Placement of the insert has been cited as a problem of this form of therapy. However, this was not borne out by the RA patients in this study. Decreased manual dexterity is therefore not a contraindication to attempting a short trial of SRAT in a patient who is experiencing difficulty in keeping the eyes adequately moist with artificial tears.

I thank Merck Sharp and Dohme Ltd for supplying the inserts, Miss $\mathrm{N}$ van Vuuren for preparing the manuscript, and the Chief Medical Superintendent, Groote Schuur Hospital, for permission to publish. Mr S Isaacs, Department of Medical Informatics, Groote Schuur Hospital, kindly performed the statistical analysis.

\section{References}

1 Lemp MA. Tear substitutes in the treatment of dry eyes. Int Ophthalmol Clin 1973; 13: 145-53.

2 Linn ML, Jones LT. Rate of lacrimal excretion of ophthalmic vehicles. Am J Ophthalmol 1986; 65: 76-8.

3 Sjögren $\mathrm{H}$. Some problems concerning keratoconjunctivitis sicca and sicca syndroma. Acta Ophthalmol (Kbh) 1951; 29: 33-47.

4 Thompson $M$, Eadie $S$. Keratoconjunctivitis sicca and rheumatoid arthritis. Ann Rheum Dis 1956; 5: 21-5.

5 Lenoch F, Brémora A, Kankova D, Streda J, Balik J. The relation of Sjögren's syndrome to rheumatoid arthritis. Acta Rheumatol Scand 1964; 10: 297-304.

6 Mody GM, Hill JC, Meyers O. Keratoconjunctivitis sicca in rheumatoid arthritis. Clinical Rheumatol 1988; 7: 237-41.

7 Gautheron PD, Lotti VJ, Le Donarec JC. Tear film break up time prolonged with unmedicated cellulose polymer inserts. Arch Ophthalmol 1979; 97 : 1944-7.

8 Bloomfield SE, Dunn MV, Miyata T, Stenzel KH, Randle SS, Rubin AL. Soluble artificial tear inserts. Arch Ophthalmol 1977; 95: $247-50$.

9 Katz IM, Blackman WM. A Soluble sustained release ophthalmic delivery unit. Am J Ophthalmol 1977; 83: 728-34.

10 Breslin CW, Katz J, Kaufman HE, Katz I. Slow release artificial tears. In: Leopold IH, Burns RF, eds. Symposium on ocular therapy. New York: Wiley, 1977; 10: 77-83.

11 Lamberts DW, Langston DP, Chu W. A clinical study of slowrelease artificial tears. Ophthalmology 1978; 85: 794-800.

12 Katz JI, Kaufman HE, Breslin C, Katz IM. Slow release artificial tears and the treatment of keratitis sicca. Ophthalmology 1978; 85: 787-93.

13 Werblin TP, Rheinstrom SD, Kaufman HE. The use of slowrelease artificial tears in the longterm management of keratitis sicca. Ophthalmology 1981; 88: 78-81.

14 Høvding G, Aasved H. Slow-release artificial tears (SRAT) in dry eye disease. Acta Ophthalmol (Kbh) 1981; 59: 842-6.

15 Wright $P$, Vogel R. Slow-release artificial tear inserts in the treatment of dry eyes resulting from the oculomucocutaneous syndrome. Br J Ophthalmol 1983; 67: 393-7.

16 Newcombe RG, Duff GR. Eyes or patients? Traps for the unwary in the statistical analysis of ophthalmological studies. $\mathrm{Br}$ J Ophthalmol 1987; 71 : 645-6.

Accepted for publication 31 December 1987. 